\title{
BOUNDEDNESS OF THE RIESZ POTENTIAL ON A COMPLETE MANIFOLD WITH NONNEGATIVE RICCI CURVATURE
}

\author{
LI JIAYU
}

(Communicated by J. Marshall Ash)

\begin{abstract}
In this paper we obtain a necessary and sufficient condition for the boundedness of the Riesz potential on a complete manifold with nonnegative Ricci curvature.
\end{abstract}

In this paper, we consider the boundedness of the Riesz potential $(-\Delta)^{-\alpha / 2}$ $(0<\alpha<n)$ on a complete Riemannian manifold with nonnegative Ricci curvature. The author [1] proved that: (1) if $V_{x}(r) \leq C_{n} r^{\beta}(\beta<n)$ then $(-\Delta)^{-\alpha / 2}$ $(0<\alpha<n)$ is never of type $(p, q)$ for any $p, q \geq 1$, and (2) if $V_{x}(r) \geq C_{n} r^{n}$ then $(-\Delta)^{-\alpha / 2} \quad(0<\alpha<n)$ is of type $(p, q)$ where $1<p<q<\infty$ if and only if $1 / q=1 / p-\alpha / n$. In this paper we improve this result and prove the following result.

Theorem. Suppose $M$ is a complete Riemannian manifold with nonnegative Ricci curvature. Then $(-\Delta)^{-\alpha / 2}(0<\alpha<n)$ is of type $(p, q) \quad(1<p, q<\infty)$ if and only if $V_{x}(r) \geq C_{n} r^{n}$ for all $x \in M$ and $1 / q=1 / p-\alpha / n$. The condition is also necessary when $1 \leq p, q<\infty$.

Proof. We only need to prove that the condition is necessary.

Assume that $(-\Delta)^{-\alpha / 2}$, is of type $(p, q)$ where $1 \leq p, q<\infty$. Then

$$
\left\|(-\Delta)^{-\alpha / 2} f\right\|_{q} \leq C_{n, \alpha, p, q}\|f\|_{p}
$$

for all $f \in L^{p}(M)$.

We set $f(y)=H(x, y, s)$ where $H(x, y, s)$ is the heat kernel of $M, x$ is a fixed point in $M$, and $s$ is a fixed positive constant.

$$
\begin{aligned}
(-\Delta)^{-\alpha / 2} f(z) & =\Gamma\left(\frac{\alpha}{2}\right)^{-1} \int_{0}^{\infty} t^{\alpha / 2-1} \int_{M} H(z, y, t) H(x, y, s) d y d t \\
& =\Gamma\left(\frac{\alpha}{2}\right)^{-1} \int_{0}^{\infty} t^{\alpha / 2-1} H(x, z, t+s) d t
\end{aligned}
$$

Using the estimate of the heat kernel [2]

$$
H(x, y, t) \geq C_{n} \cdot \frac{1}{V_{x}(\sqrt{t})} e^{-\rho^{2}(x, y) / 3 t},
$$

Received by the editors October 17, 1991.

1991 Mathematics Subject Classification. Primary 43A22, 58G10.

Key words and phrases. Riemannian manifold, Riesz potential, heat kernel.

This research was supported by NECF of China. 
one has

$$
(-\Delta)^{-\alpha / 2} f(z) \geq \Gamma\left(\frac{\alpha}{2}\right)^{-1} C_{n} \int_{0}^{\infty} t_{0}^{\alpha / 2-1} \frac{1}{V_{x}(\sqrt{t+s})} e^{-\rho^{2}(x, z) / 3(s+t)} d t .
$$

By the Bishop comparison theorem we have

$$
\frac{V_{x}(\sqrt{s})}{V_{x}(\sqrt{s+t})} \geq\left(\frac{\sqrt{s}}{\sqrt{t+s}}\right)^{n}
$$

so

$$
\begin{aligned}
(-\Delta)^{-\alpha / 2} f(z) & \geq C_{n, \alpha} \frac{1}{V_{x}(\sqrt{s})} \int_{0}^{\infty} t^{\alpha / 2-1}\left(\frac{\sqrt{s}}{\sqrt{t+s}}\right)^{n} e^{-\rho^{2}(x, z) / 3(t+s)} d t \\
& \geq C_{n, \alpha} \frac{s^{\alpha / 2}}{V_{x}(\sqrt{s})} e^{-\rho^{2}(x, z) / 3 s}
\end{aligned}
$$

$$
\left\|(-\Delta)^{-\alpha / 2} f(z)\right\|_{q} \geq C_{n, \alpha, q} \frac{s^{\alpha / 2}}{\left(V_{x}(\sqrt{s})\right)^{1-1 / q}}\left(\frac{1}{V_{x}(\sqrt{s})} \int_{M} e^{-q \rho^{2}(x, z) / 3 s} d z\right)^{1 / q} .
$$

We choose the geodesic spherical coordinates about $x$ in $M$. Since the measure of $\operatorname{Cut}(x)=0$, we may ignore it and assume $d V=\sqrt{g(\rho, \theta)} d \theta d \rho$.

$$
\begin{aligned}
\frac{1}{V_{x}(\sqrt{s})} \int_{M} e^{-\left[q \cdot \rho^{2}(x, z)\right] / 3 s} d z & =\frac{1}{V_{x}(\sqrt{s})} \int_{0}^{\infty} e^{-\left(q \cdot \rho^{2}\right) / 3 s} d V_{x}(\rho) \\
& =\frac{1}{V_{x}(\sqrt{s})} \int_{0}^{\infty} V_{x}(\rho) e^{-\left(q \cdot \rho^{2}\right) / 3 s} \frac{2 q \rho}{3 s} \cdot d \rho .
\end{aligned}
$$

Using the Bishop comparison theorem we have

$$
\frac{V_{x}(\sqrt{s})}{V_{x}(\rho)} \leq 1+\left(\frac{\sqrt{s}}{\rho}\right)^{n}, \quad \frac{V_{x}(\rho)}{V_{x}(s)} \geq \frac{1}{1+(\sqrt{s} / \rho)^{n}} .
$$

Substituting (7) into (6) yields

$$
\frac{1}{V_{x}(\sqrt{s})} \int_{M} e^{-\left[q \cdot \rho^{2}(x, z)\right] / 3 s} d z \geq \frac{2}{3} q \int_{0}^{\infty} \frac{1}{1+(\sqrt{s} / \rho)^{n}} \cdot e^{-\left(q \cdot \rho^{2}\right) / 3 s} \frac{\rho}{s} d \rho=C_{n, q} \text {. }
$$

Substituting (8) into (5) yields

$$
\left\|(-\Delta)^{-\alpha / 2} f(z)\right\|_{q} \geq C_{n, \alpha, q} \frac{s^{\alpha / 2}}{\left(V_{x}(\sqrt{s})\right)^{1-1 / q}}
$$

On the other hand, clearly one obtains [2]

$$
\|f(z)\|_{p} \leq C_{n, p} \frac{1}{\left(V_{x}(\sqrt{s})\right)^{1-1 / p}} .
$$

Substituting (9) and (10) into (1) we have

$$
s^{\alpha / 2}\left(V_{x}(\sqrt{s})\right)^{1 / q-1} \leq C_{n, p, q, \alpha}\left(V_{x}(\sqrt{s})\right)^{1 / p-1} .
$$

So

$$
\left(V_{x}(\sqrt{s})\right)^{1 / p-1 / q} \geq C_{n, p, q, \alpha} S^{\alpha / 2}
$$


If $1 / p-1 / q \leq 0$, inequality (12) could not hold for $s$ sufficiently large; therefore, we may assume $1 / p-1 / q>0$. So

$$
\begin{aligned}
& (\sqrt{s})^{n(1 / p-1 / q)} \geq C_{n, p, q, \alpha} s^{\alpha / 2} \text { for all } s>0 ; \\
& \quad \text { so } 1 / p-1 / q=\alpha / n, \text { that is, } 1 / q=1 / p-\alpha / n .
\end{aligned}
$$

Substituting (13) into (12) yields

$$
V_{x}(\sqrt{s}) \geq C_{n, p, q, \alpha}(\sqrt{s})^{n}
$$

for all $x \in M$. Equations (13) and (14) imply that the condition is necessary.

\section{REFERENCES}

1. J. Li, Gradient estimate for the heat kernel of a complete manifold and its applications, J. Funct. Anal. 97 (1991), 293-310.

2. P. Li and S. T. Yau, On the parabolic kernel of the Schrödinger operator, Acta Math. 156 (1986), 153-201.

Department of Mathematics, Anhui University, Hefei, People's Republic of China Current address: Mathematics Group, ICTP, P.O. Box 586, 34100 Trieste, Italy E-mail address: lij@ictp.trieste.it 